\title{
Cost Benefits of a Peer-Led Asthma Self-Management Program for Adolescents
}

\author{
Hyekyun Rhee, PhD, PNP1, Irena Pesis-Katz, PhD'1, and Jingping Xing, MD² \\ 1 University of Rochester Medical Center, School of Nursing, Rochester, New York \\ 2 University of Rochester Medical Center, Community and Preventive Medicine, Rochester, New \\ York.
}

\section{Abstract}

Objective-Frequent use of healthcare services associated with pediatric asthma places substantial economic burden on families and society. The purpose of this study is to examine the cost saving effects of a peer-led program through reduction in healthcare utilization in comparison to an adult-led program.

Methods-Randomly assigned adolescents (13-17 years) participated in either peer-led ( $n=59)$ or adult-led $(n=53)$ asthma self-management program. Healthcare utilization data were collected at baseline and at 3-, 6- and 9-months post-intervention. Negative binomial regression models were conducted to examine the effects of the peer-led program on healthcare utilization. Net cost savings were estimated based on differences in program costs and healthcare utilization costs between groups.

Results-Significant group differences were found in acute office visits and school clinic visits after controlling for race and socioeconomic status. The incidence rate of acute office visits was $80-82 \%$ less for the peer-led group during follow-ups. The peer-led group was 4 to 5 times more likely to use school clinics due to asthma than the adult-led group during follow-ups. The nonresearch cost of peer-led program per participant was lower than the adult-led program, $\$ 64$ vs. $\$ 99$ respectively. The net cost saving from the reduction in acute office visits and the lower program costs of the peer-led program was estimated $\$ 51.8$ per person for a 3-month period.

Conclusions-An asthma self-management program using peer leaders can potentially yield healthcare cost savings through the reduction in acute office visits in comparison to a traditional program led by healthcare professionals.

\section{Keywords}

Adolescents; Peer leaders; Camp; Healthcare utilization; Cost savings

\footnotetext{
Address all correspondence to Hyekyun Rhee. University of Rochester, School of Nursing, 601 Elmwood Ave. Box SON, Rochester, NY 14642; Office 585-276-3775; Fax 585-273-1270. hyekyun_rhee@urmc.rochester.edu..

Declaration of Interest

All authors do not have any financial and personal relationships with other people or organizations that could influence (bias) this work. We have no discloses of any affiliation with any organization with a financial interest, direct or indirect, in the subject matter or materials discussed in the manuscript (such as consultancies, employment, paid expert testimony, honoraria, speakers bureaus, retainers, stock options or ownership, patents or patent applications or travel grants) that may affect the conduct or reporting of the work submitted.
} 


\section{Introduction}

In 2009, over 7 million children under the age of 18 (9.6\%) in the United States (U.S.) reported a current diagnosis of asthma, of which nearly 2 million were adolescents (1). Asthma remains a serious problem for many adolescents and into adulthood (2). According to a CDC report (3), over 15\% of high school students reported current asthma, of whom $40 \%$ had experienced asthma attacks or episodes during the preceding 12 months. In fact, asthma attacks are as common for adolescents as for younger children (4), and near-fatal episodes are the highest among those 12-15 years of age (5).

When compared to an age matched general population of children (1-17 years) without asthma, children with asthma tend to use considerably more urgent and non-urgent healthcare services $(6,7)$. The frequent use of healthcare services associated with pediatric asthma places heavy economic burden on families and society in general. The National Surveillance Report in 2007 revealed that pediatric asthma accounted for 4.7 million physician visits, nearly 700,000 emergency department (ED) visits, and over 200,000 hospitalizations annually (8). Total indirect medical expenditures for children with asthma were $92 \%$ higher than the expenditures in those without asthma (9). Based on data from the annual National Hospital Discharge Survey and the annual National Ambulatory Medical Care Survey, the National Heart, Lung, and Blood Institute (NHLBI) estimated the annual economic costs of asthma to be nearly $\$ 21$ billion in 2011, including \$15.6 billion in direct health care costs for treating asthma and $\$ 5.1$ billion in indirect costs (e.g. lost productivity by adult caregivers) (10). Kamble and Bharmal (9) reported that annual direct medical expenditure in treating pediatric asthma in the U.S. was $\$ 6.39$ billion in 2007 U.S. dollars. It was estimated that the direct cost of asthma was $\$ 3,257$ per person annually in 2009 (11). The distribution of asthma-related expenditures showed that approximately $40 \%$ to $60 \%$ of the total medical expenditure was accounted for by office based visits and prescription medications $(6,9-11)$.

Given the high asthma morbidity and its ever increasing healthcare costs in the pediatric population, it is imperative to develop and implement effective interventions. Various interventions have been developed and evaluated to address asthma morbidity in children. Overall, many clinical trials have demonstrated that asthma self-management interventions for children are effective in improving asthma outcomes including reducing healthcare utilization such as ED visits and hospitalizations (12). Nonetheless, the extent to which the reduction of healthcare utilization is translated into cost savings is seldom investigated. To date, few interventions focus exclusively on asthma in adolescents, and data on their cost savings via changes in healthcare utilization patterns in this population are limited. We recently completed a randomized controlled study that evaluated the effectiveness of a peerled intervention in improving asthma outcomes in adolescents (13). The purpose of the current study was to examine the impact of the peer-led intervention on healthcare utilization and its associated costs in comparison to an adult-led asthma program (control group).

\section{Methods}

\section{Study Design and Intervention}

We implemented a prospective study design to examine the economic impact of a peer-led asthma self-management program for adolescents. This study was based on 9 months healthcare utilization data collected for a randomized controlled study evaluating the effectiveness of the peer-led asthma program in comparison to an adult-led asthma program. Participants were randomly assigned to either intervention or control group that received a peer-led program and an adult led program, respectively, at an asthma day camp in 2007. 
The camp was designed specifically for this study. Prior to leading the intervention group, peer leaders completed a total of 15 hours ( 5 hours/week for 3 weeks) of rigorous training sessions covering basics about asthma, psychosocial issues, asthma self-management skills and leadership skills. Peer leaders had an asthma diagnosis for at least one year and were on one or more control medications when enrolled in the study. The intervention group was divided into six small groups by age (13-14 years and 15-17 years) and gender (boys-, girlsonly groups and co-ed group), and each group composed of 8-10 adolescents was led by two peer-leaders. The control group was divided into two groups, younger (13-14 years, $n=26$ ) and older (15-17 years, $n=19)$ groups, that were led by a physician and two nurse practitioners. The content of the asthma program was comparable for both groups, focused on building both knowledge-base and skills on asthma and its self-management (e.g., basics about asthma, triggers, medications, symptom monitoring and management, asthma action plan and psychosocial issues). Follow-up data were collected from both groups at 3-, 6- and 9-months post-intervention in 2007-2008. Further details of the intervention are described elsewhere (13).

\section{Study Sample}

Eligibility criteria included ages between 13-17 years, having a diagnosis of asthma for at least one year and currently experiencing persistent asthma, as defined by the National Guidelines (14). Adolescents who had other chronic diseases (e.g., diabetes) or emotional disorders (e.g., depression) or were unable to communicate in English were excluded from the study. Participants were recruited from clinical practices and local schools in a northeastern city and its adjacent suburbs and randomly assigned to either a peer-led or adult-led group. Out of 112 adolescents who completed baseline data, 91 attended the camp (46 in the peer-led group and 45 in the adult-led group).

\section{Study Instrument and Data Collection}

Parents completed a demographic form that provided sociodemographic information of the participants including adolescents' age, gender, and race and annual gross family income as well as the parent's education. All study participants reported their asthma associated healthcare services utilization every 3 months for 9 months after the program. The healthcare services included hospitalizations, ED visits, asthma specialist visits, primary care provider (PCP) visits for worsening asthma (acute office visits) and scheduled visits (routine office visits), and school clinic visits. Adolescents indicated whether they had ever used each service and provided the number of days or times of any utilized services within the last 3 months. The healthcare utilization form was pilot tested for clarity and understanding by adolescents prior to the current study, and the reading level was $6^{\text {th }}$ grade based on Flesh-Kincaid Grade level.

Informed consent and assent were obtained from the parents and adolescents prior to data collection. Study protocol was approved by the Institutional Review Board (IRB) in a university medical center affiliated with the researchers.

\section{Data Analysis}

The demographic and baseline healthcare utilization were compared using Chi-squared test, Student's t-test and Wilcoxon rank sum test, as appropriate. The effect of intervention on the number of healthcare services was estimated by fitting negative binomial regression models. This approach was chosen due to over dispersion of the non-normally distributed count data in which the variance was larger than the mean. In addition, our outcome variables were count variables (e.g., number of visits) and each participant had the same length of observation time ( 9 months). A separate regression model was estimated for each utilization outcome (e.g., number of office visits). For each outcome, we included the participant's race, 
household annual income, and years of parents' education in the regression analyses to control for socioeconomic status (SES). We used incidence-rate ratios (IRR) to show the strengths of links of various factors with the number of healthcare services utilization measured by number of days or visits. All analyses were conducted using SAS version 9.2. An a priori alpha level of 0.10 was used for all statistical tests given the small sample size.

Subsequently, we calculated the costs incurred in implementing the program for each group. We first estimated program costs as a research project, which included peer leader payments for attending training sessions, payments for subjects for completing study questionnaires and transportation to camp in addition to all costs as a community program. Community program costs included payments for adult camp educators, camp rental fee, recreational camp activities, food, and printing materials. Based on our findings from the negative binomial models, we further estimated the economic impact of differences in healthcare utilization. We estimated net cost savings as the cost difference between the intervention and control groups associated with program implementation minus the charges difference between the two groups resulting from differences in health care utilization. We estimated the charges of each type of healthcare services using the submitted charges for in-network services and based on the $75^{\text {th }}$ percentile of all charges for the same service in the same geographic location, as submitted by multiple insurers. Specifically for office visits, we used the submitted charges for in-network level 3 office visit (CPT code 99213). We obtained the charges data from the 2008 consumers' cost estimates benchmark dataset with specification for Upstate NY (15). All costs and charges were estimated in 2008 US Dollars (adjusted when appropriate) using the Consumer Price Index inflation tables provided by the US Department of Labor (16). Most school clinics are part of schools' existing infrastructure that provides needed healthcare to all their students. We assume that the marginal variable costs due to added number of asthma-related school clinic visits would be miniscule and should not change the cost base of these school clinics (17).

\section{Results}

Table 1 provides a comparison of the peer-led and adult-led groups based on demographic characteristics including age, sex, race, income, years of asthma, years of parent education and insurance coverage and healthcare utilization at baseline. No significant group differences were found as expected by the randomization design. The majority of the sample had either public or private insurance $(96.23 \%$ in the adult-led group and $98.31 \%$ in the peer-led group).

Table 2 displays the average number of each healthcare service use over time by participants in the peer-led and the adult-led groups. Significant group differences were found in PCP visits due to worsening asthma (acute office visits) and school clinic visits at follow-up time points. No significant group differences were found in specialist visits, PCP visit for routine check-up of asthma, ED visits or hospitalizations. Multivariate regression model results, controlling for participants' age, gender, race, years of parents' education and annual family income, also suggested that there were no differences in specialist visits and PCP visits for routine check-up of asthma. Due to the small number of events within our sample, the models for ED visits and hospitalizations did not converge. Table 3 shows the results of the negative binomial regression model predicting IRRs of acute office visits from baseline to three follow-up points. The peer-led group had fewer acute office visits than the adult-led group at 3- and 9-month follow-ups, indicating that the incidence rate of acute office visits was $82 \%$ and $80 \%$ less for the peer-led group, respectively. Race and family income, and years of parent education were not significantly associated with the number of acute office visits. Table 3 also displays the results of the regression model predicting IRRs of school clinic visits due to asthma from baseline to three follow-up points after adjusting for race 
and SES. The analysis was conducted after removing four outliers. The incidence rates of school clinic visits due to asthma in the peer-led group were 3.87, 4.01, and 4.86 times greater than in the adult-led group at 3-, 6-, and 9-month follow-ups, respectively. The rate of school clinic visits in non-whites was $58 \%$ lower than that of whites. Years of parent education was negatively associated with school clinic visits.

Itemized costs for the program as a research project and the costs when the program was translated to the community are shown in Table 4. The research team organized the camp that was specifically designed for the implementation of the program, and camp related expenses listed in the Table were paid by a research grant. The total study costs were $\$ 7,955$ and $\$ 7,305$ for the peer-led program and the adult-led program respectively. Each peer leader received a total of $\$ 150$ for participating in the training sessions, and each adult educator (a physician and two NPs) received $\$ 600$ for leading the camp program. They translated to $\$ 173$ per individual in the peer-led group and $\$ 162$ per individual in the adultled group. Study costs for the peer-led group were slightly higher than those of the adult-led group primarily due to the costs incurred in paying peer leaders for training sessions. The total community (non-research) costs estimates including the camp operation expenses were $\$ 2,965$ for the peer-led program and $\$ 4,465$ for the adult-led program. The individual costs were lower for the peer-led program with $\$ 64$ per capita, assuming no financial compensation for peer leaders, compared to $\$ 99$ per capita in the adult-led program.

Table 5 summarizes the economic implications of the program. Net cost savings were calculated as the cost difference between the groups associated with the program implementation minus the costs difference between the groups resulting from acute office visits. At 3-month follow-up, compared to the adult-led program, the net cost saving from the peer-led program was $\$ 5.8$ per person which reflected $\$ 11$ more per person for the cost of the peer-led program offset by $\$ 16.8$ less per person associated with acute office visits, assuming the average cost for an office visit to be $\$ 80$ in 2008 U.S. Dollars (15). If the program was provided in a non-research setting, the net cost saving was estimated $\$ 51.8$ per person for 3 months, which was $\$ 35$ cost saving for cost of the program plus $\$ 16.8$ in saving associated with reduced number of acute office visits in the past 3 months. The 9-months follow up showed similar findings, while the 6-month follow up did not provide any differences between the two study groups.

\section{Discussion}

This study provides evidence that the peer-led self-management program can not only be less costly to implement, but also has the potential to yield healthcare cost savings in comparison to a conventional adult-led program. We found that the cost savings were primarily due to reduction in the number of acute office visits. Studies have concurred that a largest portion of asthma-related expenditures in children and adolescents are attributable to office visits and prescriptions $(6,7,9)$. Kamble and Bharmal (9) estimated that annual total expenditures associated with asthma per child was \$1,004 in 2004, and office visits accounted for the largest proportion of the expenditure estimated at \$205. Given that, the peer-led program's demonstrated ability to reduce the number of acute office visits is particularly important. When applying the 3-months cost saving per person (nearly \$52) to 2 million adolescents with asthma for a full-year period in the U.S., the economic impact of the program cannot be overstated. Our cost saving estimation may be conservative because it was based on a comparison to yet another type of intervention (i.e., adult-led program) instead of a no-treatment group. Studies have shown reductions in healthcare utilization after asthma education of any format $(12,18,19)$. Therefore, if the cost savings of the peer-led program had been assessed against the condition where no program was provided (e.g., usual care), the economic impact could have been greater. 
It is also worth mentioning that while reducing the acute office visits, the peer-led program drastically increased the likelihood of school clinic utilization due to asthma by the participants throughout the study period. School clinics are often used by students with asthma for primary and preventive health services such as medication administration, assessment or treatment of early signs of asthma symptoms. Hence, the increased use of school clinics may suggest the participants' improved asthma self-management involving adherence to treatment and symptom awareness or monitoring. Peer dynamics formed through the intervention may also have affected the use of school clinics to some extent because observing peers utilizing school clinics for asthma could increase the likelihood of the similar behavior (i.e., school clinic use) in other adolescents. It is likely that the timely and adequate use of school clinics could have prevented asthma from worsening, thus reducing the needs for acute office visits by the peer-led program participants. In general, most school clinics are an existing infrastructure that provides primary health services to students with minimal marginal costs due to added asthma-related visits. Unlike physician office visits, the services provided by school clinics are not directly billable to the students or third party payers. Therefore, additional school clinic use is not directly translated to added costs to individuals or healthcare systems. If the number of additional school clinic visits grows considerably, the school may need to increase current staffing, eventually leading to increased overall costs to the school system or taxpayers $(20,21)$. Nonetheless, we do not anticipate that the increased school clinic use by the intervention group was substantial enough for the schools to increase its staffing for their school clinics during our 9-month study period. Thus, appropriate utilization of pre-existing school clinics by adolescents with asthma appears to contribute to not only facilitating asthma selfmanagement but also minimizing economic burdens associated with acute PCP visits.

Little is known about the impact of school clinics on asthma and associated healthcare utilization. On the other hand, there is a wealth of literature that supports the positive influence of the school-based health centers (SBHCs) on health outcomes in school-age children with chronic conditions such as asthma $(17,21-23)$. The SBHC is a special type of healthcare facility primarily established and supported by the federal government to overcome barriers to healthcare access for underserved children while containing healthcare costs (24). Studies have reported that SBHCs could reduce hospitalizations, ED visits and acute outpatient visits in children and adolescents with asthma as well as opportunistic costs due to school absenteeism or parents' work loss, leading to substantial direct and indirect cost savings associated with pediatric asthma $(20,25,26)$. In the New York State, a total of 216 SBHCs are in operation in 2011, of which the majority (64\%) are located in the Metropolitan region and only $4 \%$ are in upstate small city areas (27). Given the distribution of the SBHCs, it is unlikely that our study participants from a city in upstate NY were the beneficiaries of the SBHCs. Nonetheless, this study suggests that appropriate use of traditional school clinics could also potentially lead to healthcare cost savings in adolescents with asthma. We speculate that the peer-led intervention may have improved participants' symptom awareness and self-management behavior that dramatically increased the likelihood of using school clinics in a timely and appropriate manner. It is also noteworthy that across both intervention groups, adolescents of minority backgrounds or those of parents with higher education levels were less likely to use school clinics compared to their white or lower parental-education counterparts. Underlying reasons of the differences warrant further investigation. The differing effects of either program suggest that the intervention should be tailored based on the race and SES levels of participants to maximize the positive impact of the program in each subgroup of adolescents.

In addition to the potential healthcare cost savings, we also estimated that the operation costs of a peer-led program could be lower compared to those of an adult-led program, provided that the programs were implemented in a non-research setting and adult educators were 
reimbursed at a more realistic rate than our conservative rate (\$600/day). According to our data, should a peer-led program offered as a non-research project, it could yield over $\$ 50$ cost savings for 3 months per adolescent when compared to an adult-led asthma program. In a non-research setting, monetary compensation offered to peer leaders could be waived. In our informal interviews, the peer leaders recognized being singled out as leaders and serving in such capacity as a privilege and appreciated the opportunity to foster their leadership skills and to earn credits for community service not to mention gaining asthma-related knowledge. They also believed that their experience as peer leaders could be useful in strengthening their college or job applications. Having recognized those benefits, the peer leaders stated that they would serve as leaders without financial compensation should the similar asthma program be offered in the future. Thus, we believe that peer leaders can be recruited and secured on the prospect of non-monetary benefits when the program is implemented in a non-research setting. Nonetheless, financial compensation can be important and necessary particularly in securing and retaining peer leaders of low socioeconomic status so that they can afford adequate amount of time for participating in training sessions and helping other teens with asthma. In an adult-led program, healthcare professionals provide the program as an extension of their healthcare service to be reimbursed, thus fees associated with adult educators should be factored in even in nonresearch settings. Should peer leaders be reimbursed at the rate that was offered in the current project ( $\$ 10$ per hour) for training in non-research setting, the individual costs would increase to $\$ 110$ per capita that is higher compared to $\$ 99$ per capita in an adult-led program. As such, a peer-led approach can be a more or less costly alternative to an adultled approach, depending on reimbursement decision for peer leaders, in providing selfmanagement training for adolescents with asthma.

Several limitations of the study should be considered in interpreting findings. Because of the convenient small sample, generalizability of the findings may be limited. In addition, we were unable to observe any impact of the interventions on more severe outcomes, specifically ED visits and number of hospitalizations. Our models for ED visits and hospitalizations did not converge as these events were rare in our relatively small sample. Simultaneously, reduction in acute office visits and estimated cost savings found in the peerled group could have been greater should a larger sample were involved. In addition, we did not have access to school clinics staffing and cost structure information. Personnel costs for training and supervising peer leaders were not include in estimating total costs for the peerled program as these tasks were performed by the staff who were not paid by the project funding. However, should a similar program be offered by community healthcare facilities (e.g., hospitals, clinics), marginal costs may incur in association with peer leader training and supervision as they would need to allocate staff and their time to carry out such tasks. Nonetheless, the potential costs were not factored into our estimates primarily due to the varying nature of such costs and our limited data. These marginal costs could have potentially raised the total expense of a peer-led program. Although we assume that the marginal variable costs due to added number of only asthma-related school clinic visits would not impact the cost base of these clinics, we were unable to measure it in the scope of this study. We relied on self-reports for healthcare utilization data, increasing the likelihood of recollection bias. A recent epidemiological study (28) reported fairly good agreement (83\%-97\%) between self-reported data and health insurance data on healthcare utilization. In the study, a 1-year recall period was used for less frequently occurring events such as hospitalizations and ED visits and a 1-month recall period for ambulatory office visits. The percentage of agreement for those less occurring events ranged from $91 \%$ to $97 \%$, and for office visits $83-96 \%$. Therefore, we would not anticipate that the nature of self-reported healthcare utilization data undermined the quality of our findings in a considerable way. Nonetheless, data accuracy could have been improved by obtaining prospective data, adopting a diary format in which the subjects would record their healthcare use as it occurs. 
Furthermore, this study was based on the number of healthcare service use and unable to incorporate detailed information regarding the types or levels of clinical care that was provided during each visit in estimating the costs. Future research using data from medical records or a third party payer (insurance or Medicaid) database is needed for more accurate and detailed assessment of healthcare utilization and associated costs. This study did not include data on prescription which accounts for a large proportion of medical expenditures in children and adolescents with asthma in the U.S. (7, 9, 29). Comparing prescription costs associated with school clinics versus PCP visits could have provided more comprehensive evidence demonstrating the impact of the intervention on asthma-related healthcare costs. More accurate estimation of direct and indirect healthcare cost savings of a peer-led program warrants further research employing a study design allowing advanced cost evaluation of the intervention.

Despite the limitations, this study has important implications for designing an asthma selfmanagement program for adolescents that can ultimately yield cost savings as well as adequate asthma control. Studies have consistently reported that asthma management programs could effectively address economic burdens associated healthcare utilization including hospitalization, ED visits, acute office visits $(12,30-32)$ and indirect costs associated with missed school days $(18,33,34)$, days of restricted activity $(33,34)$ and caregivers' absences from work $(34,35)$. The 2007 National Guidelines for asthma diagnosis and management acknowledge the importance of asthma self-management programs in achieving optimum asthma care and recommend that such programs need to be covered by reimbursement structures (14). Meng et al.'s (19) study echoed the importance of expanding coverage of asthma education to children as they found that providing asthma self-management education whose expense was covered by Health Management Organizations resulted in ultimate cost savings due to reduction in healthcare utilization. Because a large proportion of the healthcare costs associated with asthma is accounted for by office visits, Kamble and Bharmal (9) have advocated for interventions that could effectively target this category of healthcare utilization. In that sense, our peer-led program that was found to decrease acute office visits is of particular importance.

\section{Conclusions}

In summary, this study offers preliminary empirical evidence that a peer-led asthma selfmanagement program for adolescents could potentially lead to healthcare cost savings particularly by reducing acute office visits while facilitating appropriate use of school clinics which are considered a low cost alternative to costly conventional healthcare services. Further study is needed to examine the types of school clinic services that adolescents use to address asthma self-management needs and to improve asthma outcomes ultimately. In addition, a larger population-based study with a robust design is needed to adequately establish the cost-benefits of the intervention in the future. To address healthcare costs effectively in adolescents with asthma, this study calls for a developmentally appropriate approach appealing to adolescents' desires for autonomy and independence from authority figure as well as their particular affinity to peers. This study also suggests the potential roles of school clinics in reducing acute healthcare utilization by adolescents, which underscores the necessity of adequate asthma management training for school health personnel. Continuous, substantial increase in school clinic use by adolescents may eventually require the schools or society to increase marginal costs associated with school clinic operation to meet the needs. Therefore, the extent of ultimate cost savings by shifting adolescents' primary care related to asthma from primary care facilities to school clinics needs to be investigated in future research. 


\section{Acknowledgments}

The authors thank Dr. Michael Belyea for his statistical consultation. We also gratefully acknowledge the assistance that Judy Brasch and other research staff had provided during the project period.

This study was supported by a grant from the NIH/NINR (R21 NR009837) awarded to Dr. Hyekyun Rhee. This study protocol has been registered with ClinicalTrials.gov (Identifier: NCT01161225).

The authors are solely responsible for the content of the paper.

\section{REFERENCES}

1. Center for Disease Control and Prevention. [September 8, 2011] 2009 national health interview survey (NHIS) data . Available at: http://www.cdc.gov/asthma/nhis/09/data.htm.

2. Morgan WJ, Stern DA, Sherrill DL, et al. Outcome of asthma and wheezing in the first 6 years of life. Am J Respir Crit Care Med. 2005; 172:1253-1258. [PubMed: 16109980]

3. Brener ND, Kann L, Garcia D, et al. Youth risk behavior surveillance--selected steps communities, 2005. MMWR Surveill Summ. 2007; 56:1-16. [PubMed: 17318115]

4. Schiller, JS.; Bernadel, L. Summary health statistics for U.S. children: National health interview survey, 2002. Vol. 220. U.S. Department of Health and Human Services; Hyattsville, MD: 2004.

5. Bruzzese JM, Bonner S, Vincent EJ, et al. Asthma education: The adolescent experience. Patient Educ Couns. 2004; 55:396-406. [PubMed: 15582346]

6. Lozano P, Fishman P, VonKorff M, Hecht J. Health care utilization and cost among children with asthma who were enrolled in a health maintenance organization. Pediatrics. 1997; 99:757-764. [PubMed: 9164766]

7. Wang LY, Zhong Y, Wheeler L. Direct and indirect costs of asthma in school-age children. Prevent Chronic Dis. 2005; 2:1-10.

8. Moorman JE, Rudd RA, Johnson CA, et al. National surveillance for asthma--United States, 1980-2004. MMWR Surveill Summ. 2007; 56:1-54. [PubMed: 17947969]

9. Kamble S, Bharmal M. Incremental direct expenditure of treating asthma in the United States. J Asthma. 2009; 46:73-80. [PubMed: 19191142]

10. National Heart, Lung, and Blood Institute. Morbidity \& mortality: 2009 chart book on cardiovascular, lung, and blood diseases. 2009.

11. Barnett SBL, Nurmagambetov TA. Costs of asthma in the United States: 2002-2007. J Allergy Clin Immunol. 2011; 127:145-152. [PubMed: 21211649]

12. Coffman JM, Cabana MD, Halpin HA, Yelin EH. Effects of asthma education on children's use of acute care services: A meta-analysis. Pediatrics. 2008; 121:575-586. [PubMed: 18310208]

13. Rhee H, Belyea MJ, Hunt JF, Brasch J. Effects of a peer-led asthma self-management program for adolescents. Arch Pediatr Adolesc Med. 2011; 165:513-519. [PubMed: 21646583]

14. National Heart, Lung, and Blood Institute. Expert panel report 3: Guidelines for the diagnosis and management of asthma. Bethesda, MD: 2007.

15. FairHealth, I. [November 8, 2011] Consumer medical cost lookup. Available at: http://fairhealthconsumer.org/medicalcostlookup/.

16. Bureau of Labor Statistics. [March 29, 2011] CPI databases, tables and calculators. 2009. Available at: http://www.bls.gov/data/\#calculators.

17. Guo JJ, Wade TJ, Pan W, Keller KN. School-based health centers: Cost-benefit analysis and impact on health care disparities. Am J Public Health. 2010; 100:1617-1623. [PubMed: 20634450]

18. Wolf FM, Guevara JP, Grum CM, Clark NM, Cates CJ. Educational interventions for asthma in children. Cochrane Database of Systematic Reviews. 2003:CD000326.

19. Meng Y, Pourat N, Cosway R, Kominski GF. Estimated cost impacts of law to expand coverage for self-management education to children with asthma in California. J Asthma. 2010; 47:581586. [PubMed: 20560832] 
20. Guo JJ, Jang R, Keller KN, McCracken AL, Pan W, Cluxton RJ. Impact of school-based health centers on children with asthma. J Adolesc Health. 2005; 37:266-274. [PubMed: 16182136]

21. Adams EK, Johnson V. An elementary school-based health clinic: Can it reduce Medicaid costs? Pediatrics. 2000; 105:780-788. [PubMed: 10742320]

22. Young TL, D'angelo SL, Davis J. Impact of a school-based health center on emergency department use by elementary school students. J Sch Health. 2001; 71:196-198. [PubMed: 11393932]

23. Kaplan DW, Calonge BN, Guernsey BP, Hanrahan MB. Managed care and school-based health centers: Use of health services. Arch Pediatr Adolesc Med. 1998; 152:25-33. [PubMed: 9452704]

24. Lear JG. School-based health centers: A long road to travel. Arch Pediatr Adolesc Med. 2003; 157:118-119. [PubMed: 12580678]

25. Tai T, Bame SI. Cost-benefit analysis of childhood asthma management through school-based clinic programs. J Community Health. 2011; 36:253-260. [PubMed: 20803167]

26. Webber MP, Carpiniello KE, Oruwariye T, Lo Y, Burton WB, Appel DK. Burden of asthma in inner-city elementary schoolchildren: Do school-based health centers make a difference? Arch Pediatr Adolesc Med. 2003; 157:125-129. [PubMed: 12580680]

27. New York State Department of Health. [September/13, 2011] School-based health center fact sheet. Available at: http://www.health.ny.gov/statistics/school/skfacts.htm.

28. Yu S, Chang H, Lin M, Lin Y. Agreement between self-reported and health insurance claims on utilization of health care: A population study. J Clin Epidemiol. 2009; 62:1316-1322. [PubMed: 19403264]

29. Lozano P, Fishman P, Vonkorff M, Hecht J. Health care utilization and costs among children with asthma who were enrolled in a health maintenance organization. Pediatrics. 1997; 99:757-764. [PubMed: 9164766]

30. Teach SJ, Crain EF, Quint DM, Hylan ML, Joseph JG. Improved asthma outcomes in a highmorbidity pediatric population: Results of an emergency department-based randomized clinical trial. Arch Pediatr Adolesc Med. 2006; 160:535-541. [PubMed: 16651498]

31. Sullivan SD, Weiss KB, Lynn H, et al. The cost-effectiveness of an inner-city asthma intervention for children. J Allergy Clin Immunol. 2002; 110:576-581. [PubMed: 12373264]

32. Kelly CS, Morrow AL, Shults J, Nakas N, Strope GL, Adelman RD. Outcomes evaluation of a comprehensive intervention program for asthmatic children enrolled in Medicaid. Pediatrics. 2000; 105:1029-1035. [PubMed: 10790458]

33. Krishna S, Francisco BD, Balas EA, Konig P, Graff GR, Madsen RW. Internet-enabled interactive multimedia asthma education program: A randomized trial. Pediatrics. 2003; 111:503-510. [PubMed: 12612228]

34. Tinkelman D, Schwartz A. School-based asthma disease management. J Asthma. 2004; 41:455462. [PubMed: 15281331]

35. Georgiou A, Buchner DA, Ershoff DH, Blasko KM, Goodman LV, Feigin J. The impact of a largescale population-based asthma management program on pediatric asthma patients and their caregivers. Ann Allergy Asthma Immunol. 2003; 90:308-315. [PubMed: 12669894] 
Table 1

Baseline characteristics of participants $(\mathrm{N}=112)$

\begin{tabular}{llll}
\hline & Adult-led group $(\mathbf{n}=\mathbf{5 3})$ & Peer-led group $(\mathbf{n}=\mathbf{5 9})$ & p-value \\
\hline Age, mean \pm SD & $14.97 \pm 1.29$ & $15.32 \pm 1.36$ & 0.17 \\
Male, $\%$ & 58.49 & 55.93 & 0.78 \\
Race (non-white), $\%$ & 52.83 & 53.45 & 0.95 \\
Years of parent education, mean \pm SD & $14.62 \pm 2.60$ & $13.81 \pm 3.01$ & 0.13 \\
Gross annual income $<\$ 30,000, \%$ & 35.85 & 47.46 & 0.21 \\
Years with asthma, mean \pm SD & $9.72 \pm 4.51$ & $10.19 \pm 4.63$ & 0.59 \\
Health insurance, $\%$ & & & 0.20 \\
$\quad$ Public & 39.62 & 55.93 & 42.37 \\
$\quad$ Private & 56.60 & 1.69 & 0.48 \\
No Insurance & 3.77 & & 0.27 \\
Use of health care in the past 3 months & & $0.24 \pm 0.97$ & 0.57 \\
Number of hospitalization days due to asthma, mean \pm SD & $0.13 \pm 0.53$ & $0.34 \pm 0.86$ & 0.81 \\
Number of emergency room visit due to asthma, mean \pm SD & $0.19 \pm 0.62$ & $0.41 \pm 0.98$ & 0.80 \\
Number of asthma specialist visit, mean \pm SD & $0.30 \pm 0.70$ & $0.55 \pm 1.01$ & 0.58 \\
Number of PCP visit due to worsening asthma, mean \pm SD & $0.52 \pm 0.83$ & $0.63 \pm 0.75$ & $1.46 \pm 5.47$ \\
Number of PCP visit for routine check-up of asthma, mean \pm SD & $0.70 \pm 1.05$ & & \\
Number of school clinic visit due to asthma, mean \pm SD & $2.06 \pm 6.37$ & & \\
\hline
\end{tabular}


Table 3

Incidence rate ratios for number of acute office visits and number of school clinic visits due to asthma

\begin{tabular}{|c|c|c|c|c|}
\hline & \multicolumn{2}{|c|}{ Number of acute office visits } & \multicolumn{2}{|c|}{ Number of school clinic visits } \\
\hline & IRR (SE) & p-value & IRR (SE) & p-value \\
\hline Race (Non-white) & $1.24(0.42)$ & 0.52 & $0.42(0.20)$ & $0.06^{*}$ \\
\hline Family income & $1.10(0.15)$ & 0.48 & $1.03(0.18)$ & 0.87 \\
\hline Parent education & $0.91(0.06)$ & 0.12 & $0.74(0.07)$ & $0.001^{* * * *}$ \\
\hline Intervention vs. control (3 month) & $0.18(0.14)$ & $0.03^{* *}$ & $3.87(2.91)$ & $0.07^{*}$ \\
\hline Intervention vs. control (6 month) & $1.13(0.89)$ & 0.88 & $4.01(2.55)$ & $0.03^{* *}$ \\
\hline Intervention vs. control (9 month) & $0.20(0.20)$ & $0.10^{*}$ & $4.86(3.28)$ & $0.02^{* *}$ \\
\hline
\end{tabular}

SE: Standard Error

$0.05<\mathrm{p} \$ 0.10$

**

$\mathrm{p}<0.05$

p $<* 0.01$ 
Table 4

Summary of study costs and potential community costs associated with the program

\begin{tabular}{|c|c|c|c|c|}
\hline & \multicolumn{2}{|c|}{ Study Costs (\$) } & \multicolumn{2}{|c|}{ Community Cost Estimates (\$) } \\
\hline & Peer-led program $(n=46)$ & Adult-led program $(n=45)$ & Peer-led program $(n=46)$ & Adult-led program $(n=45)$ \\
\hline Peer leader training & 2100 & & -- & \\
\hline Camp educators & & 1800 & & 1800 \\
\hline Subject payment & 2300 & 2250 & -- & -- \\
\hline Camp rental fee & 200 & 200 & 200 & 200 \\
\hline Camp activities & 875 & 575 & 875 & 575 \\
\hline Foods & 1510 & 1510 & 1510 & 1510 \\
\hline Printing cost & 380 & 380 & 380 & 380 \\
\hline Transportation & 590 & 590 & -- & -- \\
\hline Total costs & 7955 & 7305 & 2965 & 4465 \\
\hline $\begin{array}{l}\text { Average cost per } \\
\text { person }\end{array}$ & 173 & 162 & 64 & 99 \\
\hline
\end{tabular}




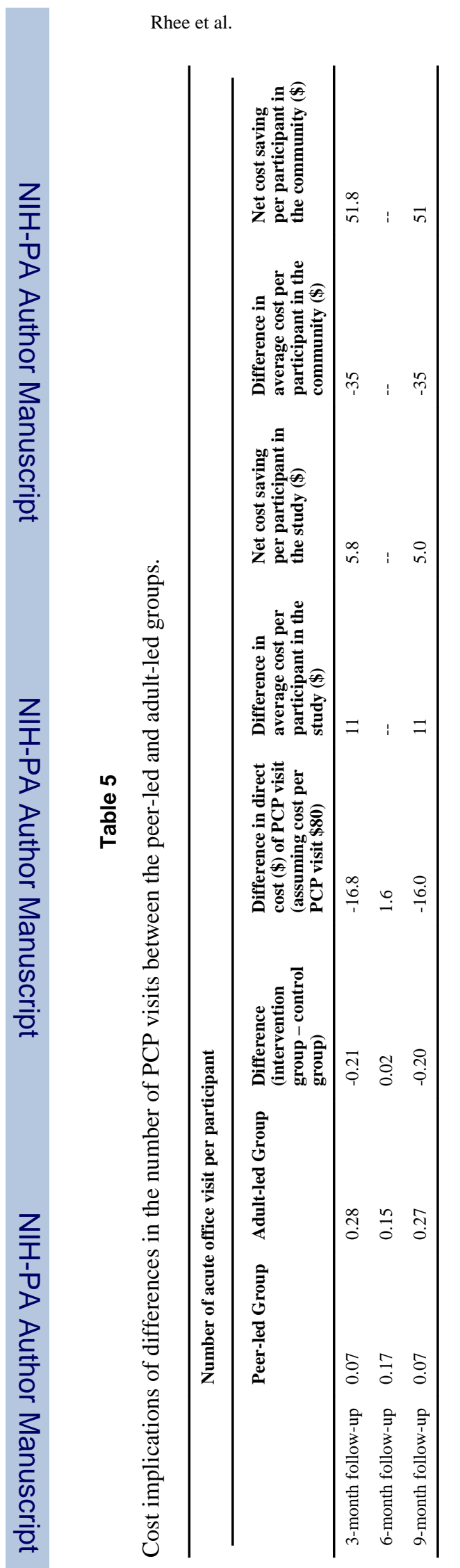

Page 15 